\title{
Optimising Syntax Generalisation for Enterprise Modelling
}

\author{
Upasana Gitanjali Singh \\ Suraj Juddoo
}

\begin{abstract}
Organisations that make use of technology for their internal and external operations, for gaining competitive advantages, are referred to as Digital Enterprises. Enterprise Modelling (EM) is the representation of a business organisation's (single or networked) structure and behaviour, to carry out analysis, (re-)design, and efficient optimisation of its operations. However, there are certain issues in the field of EM that create strategic challenges for digital enterprises currently, e.g. to determine the relationship and constraints between high level modelling and analytical modelling. A systematic literature review had been carried out to address this matter. Amongst others, this article highlights constraints that must be considered when developing process models and temporal logic. One step towards syntax generalisation of enterprise models is by viewing the enterprise from the perspective of the business services it uses and renders to its clients.
\end{abstract}

Keywords: Enterprise Modelling (EM), Digital EM, High Level Modelling, Analytical Modelling, Ontology.

\section{Introduction}

Despite the accelerating pace of changes experienced by the business sector, the factors which generate these changes tend to be rather complex. The business world of today now contains co-operation between different organisations, which eventually leads to rapid changes in business processes and activities. This also creates an intensive competitive environment, thus 


\section{Upasana Gitanjali Singh \& Suraj Juddoo}

requiring the ability to respond efficiently to rising challenges. This is a necessity for enterprises, to leverage situations into their competitive advantage. In these processes, they are gradually transformed into a globally integrated, dynamic or adaptive organisation (Jelena et al. 2015).

Enterprise Modelling (EM) is the representation of a business organisation's (single or networked) structure and behaviour, to carry out analysis, (re-)design, and efficient optimisation of its operations (Venadat 2003). In basic terms, EM entails a systematic method of analysing and modelling organisations' goals, processes, structures, products, information systems or other factors relevant to their modelling. It aims to solve business and Information Technology (IT) problems, through a holistic approach, by ensuring that the proper tools, languages and techniques are used to provide models with distinct perspectives of organizational knowledge and information systems (Jelena et al. 2015).

Throughout the years, different techniques and methods in EM have come into existence. Regardless of all the positive progresses made, a larger percentage of EM techniques and methods are essentially descriptive in nature, focusing mainly on two aspects: Function and Information. "Processbased or workflow-oriented" methods which depict an organisation's behaviour in a more detailed and accurate way have only been recently developed in the latter years (Bernus, Mertins \& Schmidt 1998). This has been an important development. In addition, to avoid the possibility of confusion amongst EM languages, due to the various proposed methods and techniques, the development of a generally acceptable EM language with specific syntax and semantics, which would act as the standard layout for all tools in the EM community is necessary. For this to be possible, it is imperative, that the relationship between high-level modelling (such as process models) and analytical modelling (such as temporal logic) be made explicitly clear (Berio \& Vernadat 1999).

Using the Systematic Literature review methodology, this research would be focused on answering the following research questions:

- How are process models and temporal logic of an enterprise developed?

- How do enterprise ontologies relate to enterprise modelling techniques? 


\section{Literature Review}

Process models as a form of high-level modelling, are graphical representations of essential processes in an enterprise. They can be used to determine the current and future business process of an enterprise as well as its value of creation (Fleischmann \& Bachinger 2014). Process models are important for carrying out process analysis, business process improvement, process-driven administration and information systems engineering or redesign in an enterprise (Nadja 2009; 1995). Process modelling can be used to provide an in-depth understanding of the workings of an enterprise and its rules, which can be used as a foundation, when critically analysing the enterprise's processes (Faisal 2010).

Process modelling methods/tools are most often categorised into two types; static methods/ tools and dynamic methods/ tools. Static methods/ tools visually depict an enterprise's processes using graphical formats by representing the processes and their individual items, with different arrows and rectangles. Dynamic methods/ tools are different, in that they function as process simulations. They dynamically represent processes using entities in the detailed physical dimension, allowing them to assess and analyse the quantitative impacts of planned processes before the processes are implemented (Yi 2008).

The key aspects of the processes which must be properly captured and represented when carrying out process modelling are the behavioural aspect and structural aspect. These two aspects enable a thorough understanding into an enterprise's structure, and the interactions between the different processes of the enterprise (Perry 2006).

An example of the process modelling method is the Role Activity Diagram (RAD), which are diagrammatic notations used in modelling the behaviour and interactions found within a process. RAD depicts all the units (roles and activities) of a process and how these units work together (Faisal 2010).

Temporal logics as a form of analytical modelling are formal frameworks used to represent logical concepts related to time (José et al. 2013). Knowledge is deemed as a very important asset in enterprises, and have therefore incorporated quality, change and knowledge management, into their essential management methods, creating an information-based management domain which enable them to compete with rivals, on the ever-changing 


\section{Upasana Gitanjali Singh \& Suraj Juddoo}

competitive market (Mach-Król 2012). The major aspects of knowledge possess temporal characteristics. With the passage of time, knowledge could become outdated, or revised, due to the emergence of new information in certain knowledge related domains. If an enterprise removes the temporal dimension from its knowledge management, it is highly possible for the enterprise's growth to stagnate, as an important element of knowledge (continuous improvement) would be lost. This makes time a critical aspect to an enterprise, when dealing with knowledge management (Mach-Król 2012).

Time is the temporal dimension that helps in providing the understanding of change. The proper use of temporal dimensions involves considering the possibility of change and its impacts (Fisher 2011). It is easy to understand and interpret such temporal reference (change) using temporal formalisms that regard time as an important variable. One example of such temporal formalism is temporal logic, which can identify relationships and interactions among activities or events, simplistically defined as 'shorter', 'longer', 'prior', 'simultaneously' and so forth. This makes it easy to arrange activities or events in a timeline, and carry out analysis on the causes of change, impacts, and its direction regardless of situations where activities or events overlap with each other (Allen 1983). The use of temporal logic in knowledge management also creates certain competitive advantages for an enterprise, as future predictions can be made from analysing historic activities or events, making the enterprise agile in adapting to the ever-changing competitive market (Fisher 2011). A 'formalism' example which supports ease and efficiency when aligning activities or events in a timeline is Allen's interval algebra (Mach-Król 2012). Allen proposed thirteen (13) foundational relations among intervals which demonstrate every possible relationship between any two-time intervals. The interpretation of Allen's intervals, requires the mapping of intervals into actual sets of numbers, with the start of an interval being entirely before the end of a previous interval. It is possible to define the relative position of two time intervals (represented by the actual sets of numbers), using one of the components of the arrangement of the thirteen foundational relations.

'Ontology' in the general sense is a method of describing a specific domain related to views or concepts, while characterising the semantics and relationships between these views or concepts. This creates a shared vocabulary for describing the fundamental knowledge of the domain, thereby enabling a clearer understanding into the domain, and facilitating the process 
of information sharing and re-use between different domains (Junhui \& Chan 2010). Enterprise ontologies (EOs), are part of an enterprise's core integration processes, which provide common vocabularies amongst the different enterprise frameworks to be integrated or modelled (Amjad \& Adalberto 2009). Jan (2008) presents the objective views or perspectives which have been used to categorised EOs over the years. These objective views or perspectives include the domain, system, planning, transactional, identification and diagnostic approaches.

The domain approach classifies an enterprise under four areas, viz. 'marketing, organisation, strategy, and activities'.

In a system approach, components are created for mapping an enterprise and its processes (Gruninger, Atefi \& Fox 2000).

The planning approach involves the developments of ontologies to be used in conceptualising different business processes. It describes an enterprise based on its human resources, assets, business units, competence, roles, tasks, privileges, knowledge and business documents (Jenz 2003).

The transactional approach incorporates the development of all ontologies that is related to the interactions within an enterprise. Four axioms are identified and defined in this approach: 'Enterprise activities'; 'Acts of coordination'; 'Business Process' and 'Ability' (Jenz 2003).

The focus point of the identification approach is the creation and filling of corporate memory resources, by conceptualising the competencies and experiences of an enterprise. This approach presents EO's which categorise an enterprise based on three constraints: 'Core business'; 'performance indicators'; and the 'cooperate memory chucks'. The EO's of the identification approach provide a clear orientation into the state or condition of an enterprise, using indicator classes such as intellectual capital markers. Other key classes found in this approach includes 'Processes, Strategies, Procedures, Activities, and KPIs (Key Performance Indicators) (Jussupova-Mariethoz \& Probst 2007).

To date, the diagnosis approach, is best represented by ontologies related to data mining. The diagnosis approach presents EOs based on the evaluation of the credit capacity of an enterprise. The EOs presented in this approach usually consists of components such as: credit data, credit requirements, assessment methodologies; and credit knowledge. This approach however, has its own limitations, as it does not have ontologies for conceptualising and making cause-effect analyses of an enterprise (Jan 2008). 


\section{Upasana Gitanjali Singh \& Suraj Juddoo}

\section{Research Methodology}

To explain the relationship between high level modelling and analytical modelling as a part of enterprise modelling, a brief systematic literature review was carried out. Unlike narrative review processes which tend to be descriptive and do not really involve a systematic conceptual analysis of literature, often results in focusing on a subset in an area of study that was chosen based on an author's selection and availability. The systematic review process on the other hand involves a systematic search of all relevant conceptualisations in available literature already made with regards to the selected topic. This means that narrative reviews, while being informative, often lead to an inclusion of selection bias, which most often cause similar studies to have diverging results and conclusions. Reducing bias by identifying, assessing and blending every single relevant study on a topic, typically involves a comprehensive and detailed plan and a search strategy derived from deductive reasoning. Most often, a systematic review process also includes the use of a meta-analysis component which makes use of statistical techniques to combine (synthesize) the data from many relevant studies into a single summary effect size or quantitative estimate (Uman 2011). The search procedure was performed utilizing both automatic and manual search approaches of books and journal papers since the 1980s. The journal articles and books are all available online of which some of them can be found in electronic databases.

Various types of articles and books were considered for research on various topics such as the business implementation of processes, business reports, conference proceedings, and experimental and empirical academic papers. The review process for all the study papers and book took place in four stages.

The first stage was to use every possible combination of the search terms. The Boolean 'OR' and the 'AND' operators were frequently used to mix the search terms together.

In the second stage, the title of each article was critically examined and any topic/ title that was outside the area of this research was rejected from all possible considerations in this research focus.

For the third stage in this process, we were left with about one hundred and twenty-seven papers, at which further selections were carried out. Sources containing editorials, prefaces, comments, news, workshops and even tutorials, were removed. 
In the fourth stage, during the last examination session of the whole corpus of papers and books, most of the related books, papers and articles were read to determine their relevance, before being used to formulate our literature review.

To determine the relevance of a paper during the screening process, certain exclusion (E) and the inclusion (I) criteria were applied. These criteria are listed below:

E1: Presentations, panels, abstracts, posters and article summaries;

E2: The publishing source was not relevant;

I1: Does the book or paper address the application of enterprise modelling?

I2: Does the book or paper have any kind of focus on the way businesses today re-engineer their business processes? and

I3: Does the paper or book consider any factors that drive a business to plan towards developing an improved business process?

By using these exclusion and inclusion criteria to determine the relevance of the books, papers and articles, a total of a forty-two books, papers and articles were finally selected from the one hundred and twenty-seven articles and books.

The sub-question (SQ) below was applied to efficiently answer the research questions, taking into consideration the point of view of other authors who have performed similar research focuses.

SQ1: What are the constraints to be considered when developing a process model and temporal logic analysis?

\section{Finding}

\section{What are the constraints to be considered when developing a process model and temporal logic analysis?}

The agility of modern-day enterprises is a crucial factor in determining the success of an enterprise. Therefore, it is important for every enterprise to have an overview of its business processes to effectively resolve issues which may arise due to deficiencies in the business process (Peter 2007). Knowledge and 


\section{Upasana Gitanjali Singh \& Suraj Juddoo}

skills in different fields are required when carrying out process modelling, as the domain encompasses the part of change, information technology and project management, while involving different stakeholders such as shareholders, suppliers, internal and external customers.

Peter (2007) explains that the business process of an enterprise must be aligned with the overall strategy of the enterprise and must introduce certain measurement tools for process performance such as strategy map and balanced scorecards. These may help in aligning the enterprise's business processes with the overall strategy. It is also important to ensure that the activities of the enterprise's internal customers are properly assessed, and rewarded using KPIs (Key Performance Indicators), which promote the bottom-line strategy of the enterprise.

Santosh, Rong and Frederick (2008) identified two factors which should be considered when developing process models and temporal logic. These factors include:

- Process scope (which is defined as a group of processes, with properly identified end-to-end characteristics, which could be used to map the inputs and outputs of the enterprise); and

- The business entity (which is defined as the key source of information associated with data models and behaviour models when discussing process scope).

Santosh, Rong and Frederick (2008) also highlighted that business entities could be linked together, if their behaviour models possess at least one common business activity. By linking business entities together, it is possible to form an 'information centric business process model'. They also stated that the addition of 'data access details' into each individual activity of the business entity, the 'information centric business process model' can be converted into an 'activity-centric model'.

De la Vara and Sánchez (2008) stated that, using business process modelling and notation (BPMN) for modelling business processes, makes process models easy to understand, especially for users of business processes such as business analysts, business managers and Information Systems (IS) engineers. This is because BPMN uses notations that are easily understandable and stipulate a standard for business modelling and its possible implementations. They further highlighted that B-SCP, is a new and innovative 
method encompassing 'business, strategy, context and processes', which verify and validate the alignment of business requirements with business strategy and business processes which complement the strategy. They however also suggest that process models created using B-SCP are less expressive than process models created using BPMN, as B-SCP makes use of Role Activity Diagrams (RADs) for modelling business processes.

The approach by Kowalkiewicz et al. (2008) requires that the understanding of business goals is a requirement for creating temporal logic. Business goals are ever-changing due to the dynamicity of the business world. Certain factors which could influence changes in business goals include:

- the availability of resources;

- reformation of business practices; and

- the implementation of new business compliance directives issued by government bodies.

According to Kowalkiewicz et al. (2008), process models are developed and implemented for different individual process types and might result in the deviation of process instances from the behaviour model when executed. They also highlight the concern that developers of process models often do not possess enough knowledge of the relevant processes, during the design phase. This is because process knowledge is part of an enterprise's corporate skill set, and can only be attained through experience. As a result, process knowledge of operations is mainly held by the enterprise's internal customers, who play certain roles in an area of the enterprise's overall operations.

Mike (2008) stated that business protocol descriptions are necessary when discussing change management. He highlighted that protocols evolve over time because of new and emerging systems, strategies, policies and quality requirements. He further went on to describe two types of protocol evolution, viz.:

- Static protocol evolution: this refers to the modification of protocol definitions by stipulating several change processes which enables current protocols to gradually transform, without the need for redescribing the protocol all over again; and

- Dynamic protocol evolution: this refers to the complete replacement of existing protocols still in execution, with a totally new protocol. 


\section{Upasana Gitanjali Singh \& Suraj Juddoo}

This usually requires the use of mechanisms to migrate and ensure that the running instances under the old protocol conforms with the requirements of the new protocol.

He further highlighted that an enterprise's service change can be distinguished into two categories:

- shallow service changes: these are changes strictly limited to the recipients (clients) of the service; and

- deep service changes: these extend beyond the recipients of the service and might even affect the entire value chain.

\section{Discussion}

In our literature review, we have highlighted several characteristics of process modelling. The illustration of essential process steps in a graphically represented format, must represent the true reflection of a business process and can illustrate the general execution or workings of a business. In this discussion we follow the sequence of ontology approaches as outlined with reference to Jan (2008) above.

The domain ontology approach is also described by Uschold et al. (1998), who classify an enterprise under the same four areas as Jan (2008), viz.: 'marketing, organization, strategy and activities'. The approach contains five vocabulary segments respectively: Meta-ontology vocabularies, planning vocabularies, an enterprise's structure vocabularies, strategy definition vocabularies, and marketing processes vocabularies. Different vocabularies segments of this approach can be used for process modelling, as they possess characteristics of process modelling. Similarly, they can also be used for temporal logic as they posses characteristics of temporal logic. Mike (2008) provided an explanation on enterprise services, as he highlighted that they are subjected to continuous changes, improvements and improvisations, thereby causing 'services' which are also a term under the marketing processes vocabularies that possesses a temporal characteristic which groups it under temporal logic. He however classified change into two categories of shallow and deep, stating that the deep service changes affect the entire value chain of the enterprise, which involves the supplier, shareholders, internal and external clients of the enterprise, 
thereby also giving 'services' a characteristic of process modelling, which 'can illustrate the general execution or workings of a business'. Hence, services can also be categorised under process modelling. This is highlighted as the first point of interception between process modelling and temporal logic.

The system ontology approach creates components for mapping the structure of an enterprise and its activities. The system approach requires the enterprise to be regarded as a group of subsystems. An example of such an approach is the Toronto Virtual Enterprise ontology, which characterises enterprise ontology integration, based on the following constraints: resource, action, goals, services, external relations, occurrence, product. Kowalkiewicz et al. (2008) highlighted that business goals are ever-changing due to the dynamic conditions of the business world. As we earlier stated, one major characteristic of temporal logic is that it changes with time. Business goals can be categorised under temporal logic. and by extension, the system ontology approach which characterises 'goals' as one of its ontology constraints can also be categorised under temporal logic.

The planning approach of enterprise ontology which involves the development of ontologies situated for the conceptualization of numerous business processes, could be considered as a process modelling suited approach, as it possesses one of the characteristics of process models. 'It must represent the true reflection of the business process'. Process scope is defined as a group of processes aligning with the application of the planning approach and can be categorised under process modelling.

The transactional approach incorporates the development of all ontologies that is related to the interactions within an enterprise, identifying four axioms, viz.: 'Enterprise activities'; 'Acts of coordination'; 'Business Process'; and 'Ability' (Dietz 2006). The transactional approach can be classified under process modelling, as it exhibits three characteristics of process modelling. 'It must represent the true reflection of a business process', which is illustrated by the Enterprise activities axiom (tasks successfully carried out due to the competence of the actors performing certain roles); it 'can illustrate the general execution or workings of a business', which is illustrated by the Act of coordination axiom (procedures of certain behavioural pattern known as transacttions); and the Business process axiom (the composition of transactions). 


\section{Upasana Gitanjali Singh \& Suraj Juddoo}

BPMN aligns with the implementation of the enterprise activity axiom of the transactional ontology approach as it is used for modelling business processes, while B-SCP, which verifies and validates the alignment of business requirements with business strategy and business processes which complements the strategy, aligns with the implementation of the Act of coordination axiom and the business process axiom of the transactional ontology approach. Hence, we determine that by extension, the BPMN and BSCP described by José (2008) can both be categorised under process modelling.

The identification ontology approach focuses on the creation and filing of corporate memory resources. In this approach, it is important to conceptualize the capabilities (competences) and experience of the enterprise. The experience and capabilities of an enterprise change with time and can be influenced by several factors of which organisation strategy and policies from external bodies such as government agency are a part of. As a result, we can categorise the identification ontology approach under temporal logic. An explanation of business protocol description which highlights that protocols evolve over time because of new and emerging systems, strategy, policies and quality requirements, aligns with the categorisation of the ontology identification approach and can also be categorised under temporal logic (Mike 2008). In order words, business protocol descriptions have temporal characteristics since they change and evolve with time.

The diagnosis ontology approach comprises of ontologies related to data mining methods. Data is a necessity when carrying out data mining, and only historical data within a specified time-frame can be extracted for analysis. Data models, consisting of similar or varying data of a specific entity are developed when carrying out data mining (Fayyad, Piatetsky-Shapiro \& Smyth 1996) as such data can be said to be of a temporal nature, since it changes with time. Hence, it can be classified under temporal logic. A business entity which is defined as the key source of information associated with data models, aligns with the use of the diagnosis ontology approach and by extension can be categorised under temporal logic. The two factors highlighted are: process scope; and the business entity, based on their characteristics, and can be categorised under process modelling and temporal logic respectively (Santosh, Rong \& Frederick 2008). 


\section{Conclusion and Future Research}

Using the Systematic literature review approach, we have been able to highlight seminal aspects which must be considered when developing process models and temporal logic, derived from existing research contributions made by different authors, also indicating constraints. These factors include: process scope, business entity, business goal, modelling methods, business protocols and business services.

We have been able to identify business services, as the relationship between high level modelling (Process models) and analytical modelling (Temporal logic). Business services not only involve the entire value chain of the enterprise (suppliers, stakeholders, internal and external clients). They also involve processes with temporal characteristics, as these evolve over time.

We therefore hypothesise that one step towards syntax generalisation of enterprise models, is by viewing the enterprise from the perspective of the business services it uses and renders to its clients. This would not only help in explicitly illustrating the business processes involved in the generation and provision of the service to the clients (both internal and external), and illustrating the process flow of the service within the different departments of the enterprise, but also allows for the proper describing of the role each department plays in ensuring the delivery of the service to the clients. It also allows for the factoring in of events and activities which occur due to the provision, improvement, improvisation and change of services provided. As such they could be properly mapped in a timeline, for better analysis of the enterprise competence, customer satisfaction and the effectiveness of the business strategy implemented by the enterprise.

This hypothesis however is open to criticism, as the resources used in this research was strictly limited to existing research papers, articles and books, by different authors in the field of enterprise modelling. No practical experiments were carried out, nor was the hypothesis tested on any enterprise to confirm its accuracy. But this was not the objective of this study.

Against this background though, practical studies may be carried out concerning the different ontologies of enterprise modelling as identified and analysed in this study. This may provide an opportunity to develop fit-for purpose enterprise modelling in African contexts, which are often different from the contexts in which the existing ontologies were developed. Such empirical research could importantly contribute toward the improvement of 


\section{Upasana Gitanjali Singh \& Suraj Juddoo}

efficiency and effectveness of enterprises in African contexts. This would also open the possibilities for further hypotheses, that might be implemented and tested in actual business systems and enterprises.

\section{References}

Abadi, A., H. Ben-Azza, S. Sekkat \& E.M. Zemmouri 2016. An Ontologybased Framework for Virtual Enterprise Integration and Interoperability. IEEE XPlore Digital Library. Available at: https://doi.org/10.1109/EITech.2016.7519607

Allen, J.F. 1983. Maintaining Knowledge about Temporal Intervals. Communications of the ACM 26, 11:832 - 843.

https://doi.org/10.1145/182.358434

Amjad, U. \& C. Adalberto 2009. Enterprise Ontologies for Planning and Integration of Business: A Pragmatic Approach. IEEE XPlore Digital Library. Available at:

https://ieeexplore.ieee.org/document/4804719?denied=

Berio, G. \& F. Vernadat 1999. New Developments in Enterprise Modelling using CIMOSA. Computers in Industry 40: 99 - 114.

https://doi.org/10.1016/S0166-3615(99)00017-2

Bernus, P., K. Mertins \& G. Schmidt 1998. Handbook on Architectures of Information Systems. Berlin: Springer-Verlag.

https://doi.org/10.1007/978-3-662-03526-9

de la Vara, J.L. \& J. Sánchez 2008. Improving Requirements Analysis through

Business Process Modelling: A Participative Approach. In Abramowicz,

W. \& D. Fensel (eds.): Business Information Systems. Proceedings of the $11^{\text {th }}$ International Conference, BIS 2008, Innsbruck, Austria, May 5-7, 2008. Berlin, Heidelberg: Springer Verlag.

Dietz, J. 2006. Enterprise Ontology Theory and Methodology. Berlin, Heidelberg: Springer-Verlag. https://doi.org/10.1007/3-540-33149-2

Faisal, A. 2010. A Method for Linking Business Process Models and System Models. IEEE XPlore Digital Library. Available at:

https://ieeexplore.ieee.org/document/5561306

Fayyad, U., G. Piatetsky-Shapiro \& P. Smyth 1996. From Data Mining to Knowledge Discovery in Databases. AI Magazine 17,3: 37.

Fisher, M. 2011. An Introduction to Practical Formal Methods Using Tempo- 
ral Logic. Chichester: Wiley.

https://doi.org/10.1002/9781119991472

Fleischmann C. 2015. A Tangible Modelling Interface for Subject-Oriented

Business Process Management. In Fleischmann A., W. Schmidt \& C.

Stary (eds): SBPM in the Wild. Springer, Cham.

https://doi.org/10.1007/978-3-319-17542-3_8

Gruninger, M., K. Atefi \& M. Fox 2000. Ontologies to Support Process

Integration in Enterprise Engineering. Computational and Mathematical

Organization Theory 6,4: 381 - 394.

https://doi.org/10.1023/A:1009610430261

Jan, A. 2008. Enterprise Ontology - Diagnostic Approach. IEEE XPlore Digital Library. Available at:

https://doi.org/10.1109/HSI.2008.4581489

Jelena, Z. et al. 2015. Advanced Enterprise Modeling. Bussiness Information Systems Engeneering 57,1: 1-2.

Jenz, D. 2003. Better Alignment of IT with Business. The Ontology-based Approach. Available at:

http://www.bpiresearch.com/WP\%20BPMAlignment.pdf

José, I.R., M.C. Gregorio, B. Roberto \& C. Manuel 2013. Temporal Logics for Phylogenetic Analysis via Model Checking. IEEE Xplore Digital Library Available at:

https://ieeexplore.ieee.org/document/6573960

Junhui, Y. \& H. Chan 2010. Research on Ontology Semantic Retrieval for Enterprise Metadata. Available at: 10.1109/FSKD.2010.5569682.

Jussupova-Mariethoz, Y. \& A. Probst 2007. Business Concepts Ontology for an Enterprise Performance and Competences Monitoring. Computers in Industry 58: 118 - 129.

https://doi.org/10.1016/j.compind.2006.09.008

Kowalkiewicz, M. et al., 2008. Models, Weak Dependencies in Business Process. In Abramowicz, W. \& D. Fensel (eds.): Business Information Systems. Proceedings of the $11^{\text {th }}$ International Conference, BIS 2008, Innsbruck, Austria, May 5-7, 2008. Berlin, Heidelberg: Springer Verlag. Mach-Król, M. 2012. Perspectives of Using Temporal Logics for Knowledge Management. In Proceedings of the Federated Conference on Computer Science and Information Systems. Available at:

https://annals-csis.org/proceedings/2012/pliks/17.pdf

Mike, P.P. 2008. The Challenges of Service Evolution. In Z. Bellahsène \& M. 
Léonard (eds.): Advanced Information Systems Engneering; CAiSE 2008. Berlin Heidelberg: Springer-Verlag.

Murdoch, J. \& J.A. McDermid 2000. Modelling Engineering Design Processes with Role Activity Diagrams. Transactions of the SDPS. Available at:

https://pdfs.semanticscholar.org/9ff6/645c0262df520d22a3490745870c6 7dbe0d5.pdf

Nabil, L. \& K. Chérif 1995. Maintaining Temporal Consistency of Multimedia Documents. Available at:

https://www.academia.edu/26850988/Maintaining_temporal_consistenc y_of_multimedia_documents

Nadja, D. \& D. Talib 2009. A Process-Oriented Modelling Technique. IEEE Xplore Digital Library. Available at:

https://doi.org/10.1109/UKSIM.2009.58

Padmanabban, V., A. Sattar, A.K. Pujari \& C. Goswamy 2000. Temporal Reasoning: A ThreeWay Analysis. IEEE Xplore Digital Library. Available at: https://ieeexplore.ieee.org/document/856600

Papazoglou, P.P. 2008. The Challenges of Service Evolution. In Bellahsène, Z. \& M. Léonard (eds.): Proceedings of the Advanced Information Systems. 20 $0^{\text {th }}$ International Conference. CAiSE 2008 Montpellier, France, June 16-20, 2008. Berlin, Heidelberg: Springer Verlag. Available at:

http://citeseerx.ist.psu.edu/viewdoc/download;jsessionid=4F7D626E87D 0381AE0725831C74E2FF0?doi=10.1.1.182.7663\&rep=rep1\&type=pdf

Perry, S. 2006. When is a Process Model Not a Process Model - A Comparison between UMLand BPMN. Stevenage.

https://doi.org/10.1049/ic:20060652

Peter, W., V. de B. Joachim, W. Jurgen \& D. Dirk 2007. The Process-Oriented Organisation: A Holistic View. In: Business Process Management. Berlin Heidelberg: Springer-Verlag.

Santhosh, K., L. Rong \& Y.W. Frederick 2008. On the Duality of InformationCentric and Activity Centric Models of Business Processes. In Bellahsène, Z. \& M. Léonard (eds.): Proceedings of the Advanced Information Systems. 20 International Conference. CAiSE 2008 Montpellier, France, June 16-20, 2008. Berlin, Heidelberg: Springer Verlag.

Uman, L.S. 2011. Systematic Reviews and Meta-analyses. Journal of the Canadian Academy of Child and Adolescent Psychiatry 20,1: 57-90. 
Uschold, M., M. King, S. Moralee \& Y. Zorgios 1998. The Enterprise Ontology. Available at: https://doi.org/10.1017/S0269888998001088

Venadat, B.F. 2003. Enterprise Modelling and Integration: From Fact Modelling to Enterprise Interoperability. s.l.:s.n.

Willaert, P., J. van den Bergh, J. Willems \& D. Deschoolmeester 2007. The Process-Oriented Organisation: A Holistic View. In Alonso, G., P. Dadam \& Michael Rosemann (eds.): Business Process Management. Proceedings of the $5^{\text {th }}$ International Conference, BPM 2007, Brisbane, Australia, September 24 - 28, 2007. Berlin Heidelberg: Springer Verlag.

Xijuan, L., R. David \& Y. Zhonghai 2010. Ontology-based Knowledge Modelling and Reuse Approach in Product Redesign. IEEE. Available at: http://toc.proceedings.com/09097webtoc.pdf

Yi, X. 2008. Process Modelling and Simulation Based on Extended UML Activity and GPSS. Available at: 10.1109/ICAL.2008.4636678

Upasana Gitanjali Singh School of Management, IT and Governance University of KwaZulu-Natal Singhup@ukzn.ac.za

Suraj Juddoo Middlesex University Mauritius Mauritius s.juddoo@mdx.ac.mu 